\title{
Guidelines on the cost-effectiveness of larval control programs to reduce dengue transmission in Puerto Rico
}

\author{
K. John McConnell ${ }^{1}$ and Duane J. Gubler ${ }^{2}$
}

ABSTRACT Objective. In the past 20 years, the emphasis for avoiding dengue epidemics has focused on larval control of Aedes aegypti, the principal mosquito vector of dengue viruses. A general consensus is that mosquito larval control holds the best promise for reducing dengue epidemics, although its actual effectiveness is still unknown and subject to a great deal of uncertainty. The objective of this research was to assess the cost-effectiveness of emergency larval control programs for reducing dengue transmission in the Caribbean island of Puerto Rico and to develop guidelines to help choose between carrying out a rapid-response, emergency larval control intervention and not conducting such an intervention.

Design and Methods. Data on dengue transmission and its likely impacts in Puerto Rico were used as a case study to develop intervention guidelines. A distribution of economic impacts was simulated using available data on disease rates and economic variables, including the costs of treating patients and the opportunity costs represented by lost wages. Successful larval control interventions were assessed by determining two parameters: (1) the costs of an intervention and (2) the expected reduction in the reported case rate of dengue. In addition, we examined how these guidelines would change with an early warning system that provides information on a possible outbreak of dengue.

Results. In Puerto Rico, larval control programs that are expected to reduce dengue transmission by $50 \%$ and cost less than US\$2.50 per person will be cost-effective. Programs that cost more per person but that further reduce transmission are still likely to be cost-effective. Having an early warning system, even one that provides a low level of accuracy, can extend the range of larval control programs that are cost-effective. For example, with an early warning system, a larval control program that reduces dengue transmission by $50 \%$ and that costs less than US\$ 4.50 per person would be expected to be cost-effective.

Conclusions. Guidelines such as the ones that we developed for Puerto Rico can be useful to public health authorities in helping to decide whether or not to spend resources for a larval control program to reduce dengue transmission. The range of larval control interventions that are cost-effective can be increased by having an early warning system that provides even a small amount of information regarding possible outbreaks.

Key words Dengue, mosquito control, disease outbreaks, epidemiologic measurements, cost-benefit analysis.

1 Oregon Health \& Science University, Department of Emergency Medicine, Portland, Oregon, United States of America. Send correspondence to: K. John McConnell, Department of Emergency Medicine, Oregon Health \& Science University, 3181 Sam Jackson Park Road, Mail Code CR114, Port- land, Oregon 97239, United States of America; telephone: (503) 494-1989; e-mail: mcconnjo@ohsu.edu 2 Centers for Disease Control and Prevention, National Center for Infectious Diseases, Division of Vector-Borne Infectious Diseases, Fort Collins, Colorado, United States of America.
In developing countries, the lack of a closed water system and adequate refuse disposal has often resulted in a proliferation of water containers that are ideal larval habitats for Aedes ae- 
gypti, the mosquito primarily responsible for the transmission of the viruses that cause dengue fever. As a consequence, epidemics of dengue can occur throughout Southeast Asia, the Indian Subcontinent, the Pacific islands, Central America, South America, the Caribbean, and parts of Africa (1). Dengue fever has an economic impact of the same order of magnitude as diseases such as malaria, tuberculosis, and hepatitis $(2,3)$.

Unfortunately, traditional methods of insecticide spraying to control adult mosquitoes have little or no effect on dengue transmission. In order to reduce the transmission of dengue, Ae. aegypti densities must be reduced to low thresholds by emptying, cleaning, treating, and removing containers and sites where eggs are laid. This process is known as larval control.

Although there is a consensus among dengue experts that mosquito larval control can be successful in preventing epidemic dengue transmission, it is generally true that such efforts do not guarantee against the possibility of an epidemic (4-8). Factors such as the level of community participation, mosquito densities, climatic conditions, and population herd immunity can all contribute to the failure of larval control interventions. In addition, although different types of larval control campaigns have been tried in a variety of locales, there is very little reliable information about what such campaigns cost.

Therefore, there are at least two challenges to providing convincing recommendations for mosquito abatement in order to limit dengue transmission. First, epidemics may be infrequent, and the cost of a larval control campaign may be unknown. Therefore, it may be difficult to say with confidence what the expected economic benefits of larval control may be. Second, a larval control campaign offers no guarantee against a possible epidemic, and interventions may not reduce disease transmission enough to avoid epidemics. In other words, resources may be expended, but the intervention may have no effect on the final result. The possibility of this lack of success in health interventions is quite real but is rarely addressed in most policy analyses.

A public health official faced with the decision of whether or not to engage in larval control may find such uncertainty particularly unsettling. The aim of this paper is to provide some guidelines about the cost-effectiveness of larval control, taking these uncertainties into consideration. By making some reasonable assumptions, we can provide some insight as to what a cost-effective program might look like. In addition, these methods provide some measure of the value of having an early warning system that provides information on a possible outbreak of dengue-even when that system is not $100 \%$ accurate.

We use data on dengue transmission and its likely impacts on the Caribbean island of Puerto Rico as a case study to develop intervention guidelines. In addition, we examine how an early warning system changes these guidelines. The presence of the Centers for Disease Control and Prevention (CDC) laboratory in the city of San Juan, Puerto Rico, has resulted in warnings in years when islandwide epidemic transmission was likely, particularly in 1986, 1994, and 1998. Since we do not have data that allow us to observe the implementation of warnings at the municipal level, we propose a simple early warning signal, and we evaluate the extent to which an early warning may result in more expensive larval control efforts still being cost-effective.

We perform the cost-effectiveness analysis through a mathematical simulation model. In using a simulation model, we take available data to build estimates of disease transmission and its associated costs. The final estimate of costs is based on a number of assumptions. The advantage of using such a mathematical model in this analysis is that it allows us to ask a number of "what if" questions without observing their actual implementation in a real-world setting. In this paper, we use modeling to examine a number of different larval-source-reduction scenarios and their associated costeffectiveness. Models have been used previously in the study of dengue to examine the effect of biological and climatic changes in the vector, most notably and exhaustively in a model of Ae. aegypti and dengue transmission by Focks et al. (9-11). Our model does not incorporate these biological dynamics but instead attempts to capture the range of costs that are associated with dengue and with larval control programs.

The interventions under consideration in this paper are assumed to be in the form of emergency mosquito larval control campaigns. Such programs entail intensive and aggressive efforts led by government agencies that are aimed at containing incipient epidemics. Radio, television, and newspaper announcements are used to inform the community of the potential for increased dengue transmission and to encourage residents to remove, empty, or cover potential larval sources. A government-led, targeted source reduction effort complements this activity, with emphasis on removing the most productive larval habitats and treating others with Abate $^{\circledR}$ (temephos) or another insecticide.

This study does not advocate for emergency vector control in preference to long-term, community-owned programs of larval control. The motivation, instead, is to provide a framework for assessing the cost-effectiveness of intensive, emergency larval control that might be used in an attempt to avoid epidemic levels of dengue.

\section{MATERIALS AND METHODS}

We are interested in comparing the expected costs of two policies: (1) no intervention and (2) an intervention with some fixed cost and indication of effectiveness. We recognize that the costs associated with both policies are uncertain, and therefore both policies may best be represented as probabilistic distributions. We first discuss the notation for and estimation of the distribution of costs for a policy of no intervention. We follow with a discussion of the distribution of costs when larval control is implemented. 
TABLE 1. Assumptions used to estimate costs associated with cases of dengue in Puerto Rico ${ }^{\mathrm{a}}$

\begin{tabular}{|c|c|c|c|}
\hline Parameter & Value & Range & Reference \\
\hline Number of total cases for every reported child (ages $0-17 \mathrm{yr}$ ) & 10 & $6-14$ & 2,3 \\
\hline Fraction of cases that are adults & 0.68 & $0.63-0.73$ & 2,3 \\
\hline Fraction of reported cases that are hospitalizations & 0.135 & $0.08-0.24$ & $2,3,12,^{c}$ \\
\hline Number of total hospitalizations for every reported hospitalization & 2.4 & $2.1-3.2$ & $2,3,12$ \\
\hline Number of total clinical visits for every reported clinical visit & 4.8 & $4.2-6.4$ & 2,3 \\
\hline Workdays lost from hospitalization & 14 & $10-18$ & $2,3,15$ \\
\hline Workdays lost from mild illness & 4 & $2-6$ & $2,3,14,15$ \\
\hline Percent of adult men employed & 90 & $80-95$ & 17 \\
\hline Percent of women employed & 80 & $80-95$ & 15,17 \\
\hline Percent of mothers employed & 22 & $15-29$ & 15,17 \\
\hline Daily wage, men (US\$) & 74 & $58-81$ & $14,15,17$ \\
\hline
\end{tabular}

a Costs reflect adjustments for inflation to the year 2000, based on the year that the original estimates were published.

${ }^{b} \mathrm{NA}=$ not applicable; we assume that half of infections occur in men and half in women.

${ }^{c}$ An additional resource on the fraction of reported cases that are hospitalizations was: Rodriguez E. Dengue outbreak in Puerto Rico (1994-1995): hospitalization cost analysis [unpublished report]. Centers for Disease Control and Prevention; 1997.

\section{Costs associated with cases of dengue when there is no larval control intervention}

Let the variable $x$ refer to the costs associated with cases of dengue. We use the notation $D(x)$ to refer to the cumulative distribution of these costs for a hypothetical municipality. $D(x)$ is on the range $[0, \infty)$. There is some probability that no dengue will be transmitted in a given municipality, and therefore the costs associated with disease, $x$, will be equal to 0 . However, there is some probability of having one or more cases of dengue. In this case, $x$ will be positive, perhaps in the millions of dollars for a community with 100000 people during a particularly bad outbreak.

This distribution, $D(x)$, can be estimated based on available data on the frequency distributions of reported cases and on the economic costs associated with such cases. Data on reported cases in Puerto Rico in the years 19831989 were obtained from the environmental health program (Programa de Higienización del Ambiente Físico Inmediato) of the Puerto Rico Department of Health. The data are comprised of suspected dengue cases by week for each of the 78 municipalities in Puerto Rico, col- lected from the CDC, private hospitals, and health centers. We focus our attention on the period of September 1 to December 31, since this is the period of peak dengue activity, accounting for approximately $60 \%$ of all cases. Reported cases are converted to reported case rates (cases per 1000 individuals) using linear interpolations of municipality population data from the Bureau of the Census of the United States (12-14).

These data suggest that the probability of a typical municipality having no reported cases of dengue during the peak season is close to $12.5 \%$. The probability of a high reporting rate, of $3.0 / 1000$ or more, is relatively small, at $9.3 \%$. The remaining $78.2 \%$ of occurrences result in low to medium levels of dengue transmission.

Table 1 lists the assumptions and sources of data that are used to develop an estimate of the costs associated with cases of dengue in Puerto Rico. In following with previous studies of dengue $(2,3)$, we assume that each reported case of dengue represents a number of unreported cases. We use this estimate of reported and unreported cases to develop estimates of total costs, which consist of total direct costs plus total indirect costs. Di- rect costs consist of the number of individuals with dengue expected to make visits to a clinic or hospital, multiplied by the costs of treating patients in a clinic or hospital $(2,3,15-18),{ }^{3}$ plus an estimated statistical value of life in order to account for the possible deaths that occur from severe forms of dengue (19). Indirect costs consist of the opportunity cost of lost work productivity from illness, and they are calculated by estimating the number of persons with dengue who are likely to miss work from illness or caring for an ill child, multiplied by their expected wages $(2,4,17,20)$.

These numbers are estimates based on reports in the scientific literature, and the final estimate, a distribution of costs, $D(x)$, is developed through a Monte Carlo simulation. First, a value for reported cases is drawn from the distribution of reported case rates. Then, based on this value and the assumptions in Table 1, estimates of the total number of cases, direct costs, and indirect costs are calculated.

\footnotetext{
Rodriguez E. Dengue outbreak in Puerto Rico (1994-1995): hospitalization cost analysis [unpublished report]. Centers for Disease Control and Prevention; 1997.
} 


\section{Costs when larval control is implemented}

The next step involves the estimation of a distribution of costs when larval control is implemented. The estimation of this distribution involves two modifications to $D(x)$ (the cumulative distribution of costs for a hypothetical municipality). One of the changes is to account for the costs associated with larval control, and the other modification is to account for the effect of larval control on disease transmission.

First, we assume that the cost of larval control is represented by $c$, a fixed per-person cost of intervention. Second, we assume that the effectiveness of larval control is known. Let $\delta$ represent the effect of this intervention, where $\delta$ is a fraction between 0 and 1 that corresponds to a reduction in cases of dengue. Given $\delta$, a value for reported case rates is drawn from the distribution of reported case rates, and that value is then multiplied by $1-\delta$.

If $\delta=0$, the intervention is considered to have no effect on the eventual number of cases of dengue, and the distribution of reported case rates is assumed to be identical to the distribution underlying $D(x)$. If $\delta=1$, the intervention is considered to be $100 \%$ effective, resulting in no cases of dengue. A $\delta$ between 0 and 1 represents a partially successful intervention.

With these two parameters, we denote $I(x+c, \delta)$ as the cumulative distribution of the costs associated with a policy with an intervention that costs $c$ per person and reduces the number of cases of dengue by $(100 \times \delta) \%$.

For large values of $c$ and small values of $\delta$, we should expect that expected total costs of the intervention policy will outweigh a policy of no larval control; the intervention will have significant costs with little effect on dengue transmission. On the other hand, if $c$ is small and $\delta$ is large, this represents a program with small costs that is successful at reducing dengue transmission, and expected total costs of larval control may be smaller than the expected costs of no intervention.

By fixing the intervention $\cos t c$, it is possible to develop an estimate of the minimum level of disease reduction $\delta^{*}$ below which a policy of emergency larval control is likely to result in smaller total costs than a policy with no intervention. To provide guidelines for appropriate use of larval control for dengue in Puerto Rico, we are interested in determining the minimum $\delta$ from samples from two distributions: $D(x)$ (outcomes with no intervention) and $I(x+c, \delta)$ (outcomes with an intervention).

Briefly, the following algorithm finds an estimate of $\delta^{*}$ by picking an initial value of $c$ and $\delta_{0}$ and then creating a new distribution that represents the outcomes with an intervention $I(x+c$, $\left.\delta_{0}\right)$. This new distribution is compared to the distribution from $D(x)$. If the expected costs of no intervention, which are $\int_{0}^{\infty} D(x) d x$, are less than the expected costs of the intervention policy, which are $\int_{0}^{\infty} I\left(x+c, \delta_{0}\right) d x$, we then choose a $\delta_{1}>$ $\delta_{0}$; if not, we choose a $\delta_{1}<\delta_{0}$. A new distribution is created from $I\left(x+c, \delta_{1}\right)$. With this new distribution, we test for either $\int_{0}^{\infty} D(x) d x<\int_{0}^{\infty} I\left(x+c, \delta_{1}\right) d x$ or $\int_{0}^{\infty} D(x) d x>\int_{0}^{\infty} I\left(x+c, \delta_{1}\right)^{0} d x$, and then we adjust $\delta$ accordingly. This process is repeated until it yields a value of $\delta^{*}$ such that the expected costs of intervention are the same as no intervention, that is, $\int_{0}^{\infty} D(x) d x=\int_{0}^{\infty} I\left(x+c, \delta^{*}\right) d x$. We repeat this process for different values of $c$ until we find the bounds on cost and effectiveness ( $c$ and $\delta$ ) that result in cost savings for larval control.

\section{RESULTS}

\section{Cost-effectiveness of larval control programs}

Figure 1 shows the zones where intervention policies are acceptable in terms of the cost-effectiveness of emergency larval control programs. The vertical axis represents a measure of effectiveness of a given larval reduction program. To aid in the interpretation of this figure, we have converted the effectiveness of a given larval reduction program, $\delta$, from a fraction between 0 and 1 to a percentage, taking values between $0 \%$ and $100 \%$. A value of $0 \%$, at the bottom of the vertical, $y$ axis, represents a program that has no effectiveness, with the resulting number of cases of dengue being the same as if no interventions were used. A value of $100 \%$, at the top of the $y$ axis, represents a program that is $100 \%$ effective, resulting in no cases of dengue. An intermediate value, such as $50 \%$, represents a program that is expected to result in half of the number of dengue cases that would be seen without an intervention. On the horizontal axis we measure the cost of a given program, in terms of dollars spent per person. A program that costs US\$ 0 per person is free; one that costs US\$ 10.00 per person represents considerable expense, e.g., US\$ 100000 for a community of 10000 people.

Given these two axes and the assumptions of our model, we then separate programs into two zones: (1) programs that are cost-effective and (2) programs that are not cost-effective. The division between programs that are cost-effective and ones that are not cost-effective is indicated in Figure 1 by the heavy dark line. The shaded area to the left of the heavy dark line represents larval control programs whose combinations of cost and reduction in disease transmission are cost-effective. The white area to the right of the heavy dark line represents larval control programs whose cost and reduction in disease transmission are probably not cost-effective. We include a band of $95 \%$ confidence intervals to reflect the uncertainty in our estimates; this band is represented by the two dashed lines in Figure 1.

From the graph in Figure 1, a range of intervention possibilities can be assessed. For example, policymakers responsible for deciding whether or not a given municipality should engage in larval control should feel confident about an intervention that costs around US\$2.50 per person and is expected to reduce disease by approximately $50 \%$. On the other hand, an intervention that costs US\$ 4.00 per person and only reduces disease by around $25 \%$ might need to be reconsidered by health administrators.

The advantage of this approach is that it acknowledges the uncertainty 
FIGURE 1. Zones where emergency larval control is cost-effective, as calculated for Puerto Rico

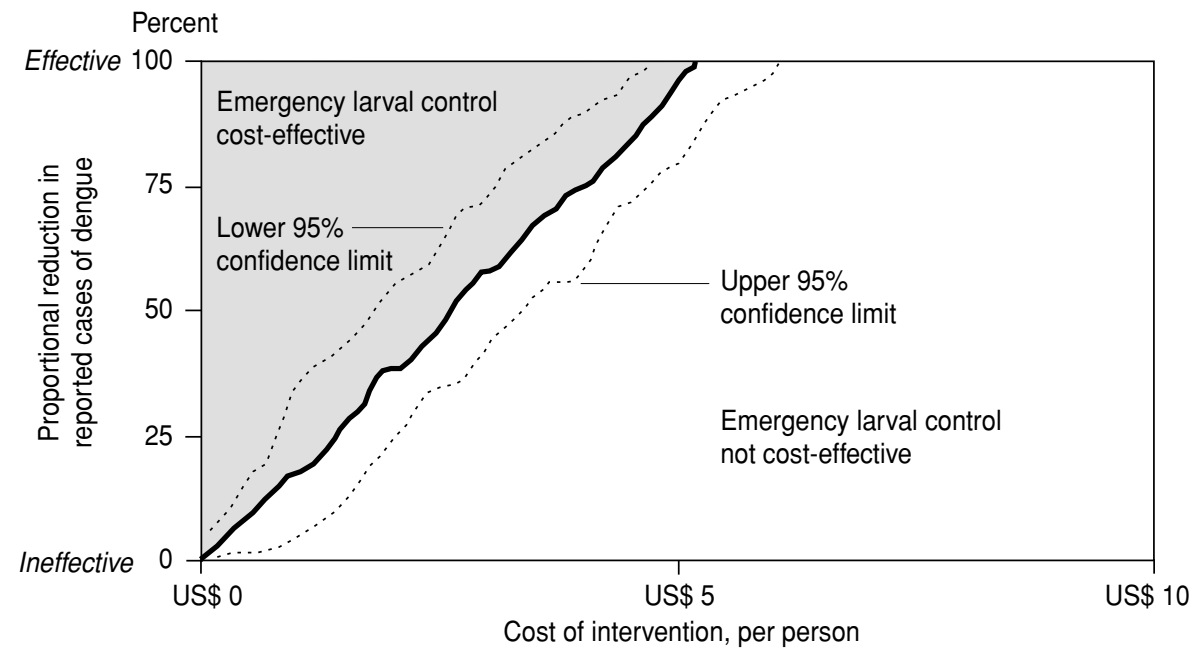

in the reported case rates of dengue and their associated costs as well as the lack of knowledge regarding the costs of larval control and its likely effect. Public health officials may have some intuition about the cost of larval control in their community and the effect of that larval control on dengue transmission. If so, the information in Figure 1 can provide them with a notion as to whether or not the implementation of such programs will ultimately produce resource savings for their community.

\section{Cost-effectiveness when there is an early warning system}

In 1983 the Centers for Disease Control and Prevention drafted a plan for an early warning system for dengue epidemics in Puerto Rico. ${ }^{4}$ The primary goal was the avoidance of major epidemics with a high incidence of dengue hemorrhagic fever. The system was to rely on epidemiologic surveillance and the seasonal nature of the disease, with epidemics occurring in the warmer and wetter fall months, generally August or September through December. Such an early warning system might reduce the impacts of dengue in the short term, while preparing the community-that

\footnotetext{
4 Gubler DJ, Casta-Vélez A. San Juan, Puerto Rico:
} Centers for Disease Control and Prevention; 1983. is, the people living in the municipality-to assume more responsibility in the long term. Ideally, a community would eventually take ownership of the joint efforts required for source reduction to be effective, providing the impetus for continued efforts to keep mosquito populations at low levels as well as continuing to educate its members about ways to empty, cover, or eliminate water containers that are productive larval habitats.

Although there is potential for using information on dengue transmission in the off-season to provide an early warning system for dengue outbreaks, it is difficult to assess the value of such warnings, since they may not be $100 \%$ accurate. In this section, we examine the effect of a hypothetical, rudimentary early warning signal on the costeffectiveness of emergency larval control programs. In particular, we show that a signal based on the number of cases observed in the summer offseason may improve the cost-effectiveness of emergency larval control programs. The implication is that some emergency larval control programs that may initially appear to be too costly to use on any occasion may be cost-saving if used in conjunction with an early warning signal.

To assess the relationship between summer and winter case rates we rely on reported case rates, using data supplied by the Puerto Rico Department of
Health from 1983-1989, with additional data on winter cases from 1982 for each of the 78 municipalities of Puerto Rico. These data are used to develop a predicted distribution of dengue cases in the peak season. The warning system is based on off-season, summer cases, defined as May 12 through July 31, or weeks 20 through 30. This definition follows a potential warning signal described by Rigau-Pérez et al (21).

We choose an off-season (May 12 through July 31) reporting rate of 0.5 / 1000 or greater to represent the point at which a hypothetical warning signal could be issued. Although this approach is not a likely (or even necessarily recommended) choice for producing real-world early warnings, it suffices for the purposes of demonstrating a method of analysis. If we define a reported case rate of 3.0/1000 or more for the period of September 1 to December 31 as "epidemic level," we can assess the accuracy of the system. Sensitivity is low $(13.9 \%)$ but specificity is reasonably high (91.9\%). However, as the following analysis will show, even an imprecise early warning system can significantly bolster the case for undertaking larval control interventions. In particular, if there is an indication that cases of dengue are likely to be high, then larval programs with higher costs may potentially become cost-effective.

The warning signal of an off-season reported case rate of $0.5 / 1000$ or greater allows for the prior distribution of reported cases to be split into two conditional distributions. One distribution is conditional on the warning signal, and the other distribution is conditional on a safe (no warning) signal. If we let $H(y)$ represent the cumulative distribution of reported cases, $y$, without information (also known as the prior distribution) and if we let ( $w$, $\sim w$ ) respectively represent the events of a warning signal and a safe (no warning) signal, then $H(y \mid w)$ represents the cumulative probability of reported cases conditional on the event of a warning signal.

Similarly, $H(y \mid \sim w)$ represents the cumulative probability of reported cases conditional on the event of a safe signal. The costs, $x$, associated with the 
distribution $D(x)$ and the distribution $I(x+c, \delta)$ are based on assumptions about the costs associated with reported cases, $y$. The relationship between the original distribution and the two conditional distributions is $H(y)=$ $p(w) H(y \mid w)+(1-p(w)) H(y \mid \sim w)$. In this equation, $p(w)$ represents the probability of an actual warning, which, based on the $0.5 / 1000$ signal, is 0.086 .

With an early warning system we have two possibilities, each of which will result in a different expected distribution of reported dengue cases. A safe (no warning) signal is represented by a new conditional distribution of cases skewed toward zero, $H(y \mid \sim w)$. A warning signal will skew the distribution toward large numbers of reported cases, $H(y \mid w)$. Based on this information, we can develop new distributions of the economic impacts of dengue for a larval control policy, $I(x+c, \delta)$, and for a policy of no intervention, $D(x)$.

Figure 2 represents the effect of a warning. As in Figure 1, the vertical axis represents the effectiveness of the given larval program, with effective programs having values closer to $100 \%$, and the horizontal axis represents the cost per person of the program. Programs that are cost-effective are those with combinations of costs and reductions in reported cases that fall in the shaded area to the left of the heavy dark line. Programs that are not costeffective are those with combinations of costs and reductions in reported cases that are in the white area to the right of the heavy dark line. The dashed lines represent a 95\% confidence interval.

Warnings of disease outbreaks substantially alter the guidelines for acceptable interventions. When no earlywarning information is available, a larval control campaign that costs more than US\$ 6.00 per person is not recommended. However, with a predicted outbreak, the same campaign is advisable, as long as the intervention can reduce disease rates by $70 \%$ or more. More costly programs may be justifiable if they have higher rates of disease reduction.

Note that the changes in policy preferences are large despite the relatively low accuracy of the early warning sys-
FIGURE 2. Zones where emergency larval control is cost-effective, as calculated for Puerto Rico, based on a warning signal from an early warning system

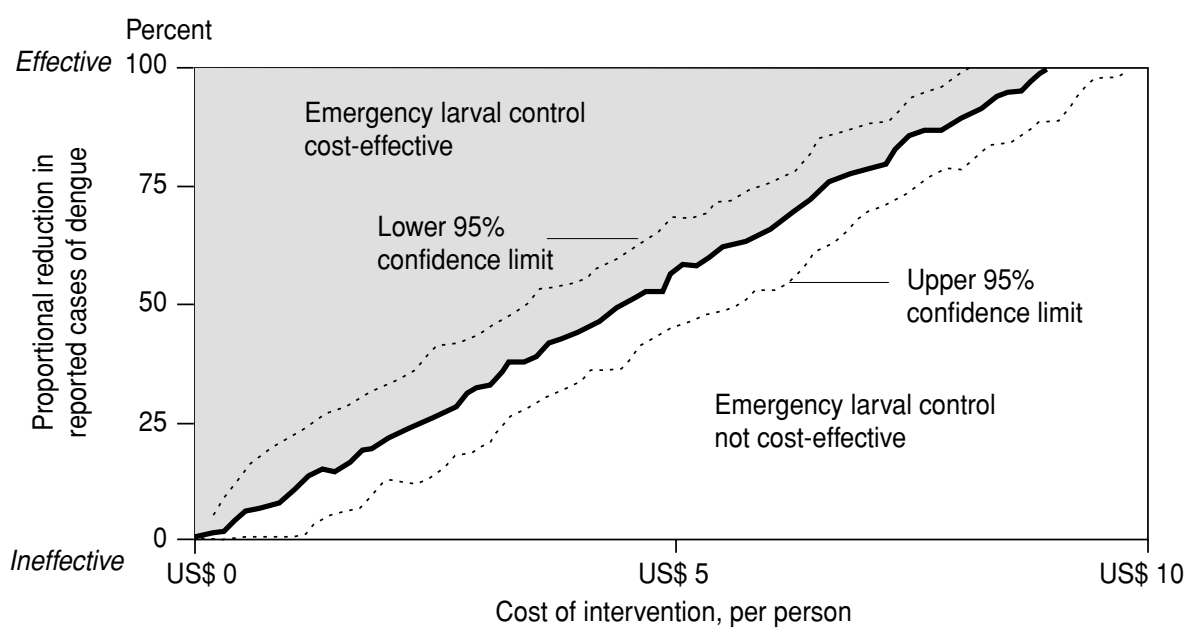

tem described herein. Anecdotal evidence suggests that government officials are typically reluctant to give approval for an intensive larval control campaign unless they are $100 \%$ sure that there is an epidemic (22). However, the guidelines in Figure 2 suggest that there may be reason to advocate for intensive larval control even when an early warning system has relatively low accuracy. Another implication of these results is that, in general, predictive information tends to have more impact when the intervention has a greater reduction in disease transmission.

\section{DISCUSSION}

This analysis suggests guidelines for the use of larval control interventions; for certain combinations of cost and rates of disease reduction these interventions are recommended. However, there are still barriers to the implementation of larval control campaigns, particularly when their use is contingent upon early warnings. Public health officials may show a reluctance to act on the basis of early warnings if they doubt the accuracy of epidemic predictions. An aim of this study is to show that a system with relatively low predictive accuracy may still provide enough information to justify larval control interventions.
This study has limitations. We used data on dengue transmission from the years 1983-1989. Higher rates of dengue transmission that have occurred in the last 15 to 20 years would suggest that we may be underestimating the costeffectiveness of emergency larval control programs. In addition, in our cost estimation, we have not included any costs associated with willingness to pay to avoid becoming ill with dengue fever. This could also result in an underestimation of the cost-effectiveness of emergency larval control. As such, the guidelines presented in this study probably represent a lower bound on cost-effectiveness.

Another limitation of our analysis is that we have avoided the specifics of larval control, which can take many forms, depending on diverse factors such as the products used, the methodology, and the quality of work and the skill of personnel involved. In addition, we have not provided guidelines about the levels that mosquito or larval densities must be reduced in order to decrease or prevent disease transmission. Focks et al. (23) have provided estimates of the reductions in larval densities required to reduce disease transmission. However, these estimates require detailed knowledge of the herd immunity of a community, the serotypes in circulation, and certain climatic variables. Thus, there are a number of interrelated factors that 
are responsible for a given antilarval program's actual success in reducing disease transmission. Rather than addressing each of these uncertainties independently, we have aggregated them into one uncertainty: the health administrator's perception of the effectiveness of a given program. The advantage of this approach is that it provides a simpler guideline for addressing the cost-effectiveness of a given larval control intervention. Since the public health official might have some notion of this uncertainty (but not the specifics of herd immunity, serotypes, climate, etc.), consideration of this single uncertainty (the effectiveness of the program) may allow for more informed decision-making. The disadvantage is that it does not draw on the evidence gathered by entomological and epidemiological studies on the best methods to reduce dengue.

There may be other effects that are factored into the decision process. For example, in some communities there may be more acceptance of epidemic outbreaks than of the inconvenience and intrusions associated with larval control efforts. In addition, political fallout might result if the warning turns out to be incorrect. One inaccurate forecast could cause the decisionmaker to lose his or her nerve as well as the public's trust. For either individuals elected to public office or government agencies subject to public scrutiny, actions taken under an erroneous forecast may have serious consequences. It is possible that an intervention based on an incorrect forecast may severely impact the credibility of future forecasts (24).

Compounding this problem is the issue of public perception. An intervention that successfully decreases a potenhemorrhagic fever: a global public health problem in the 21st century. In: Scheld WM, Armstrong D, Hughes JM, eds. Emerging infections 1. Washington, D.C.: American Society for Microbiology; 1998. Pp. 1-14.

2. Meltzer MI, Rigau-Pérez JG, Clark GG, Reiter P, Gubler DJ. Using disability-adjusted life years to assess the economic impact of dengue tial epidemic has an outcome that may be hard to distinguish from the case when the prediction was incorrect. In this case, the decisionmaker may have an incentive to wait for the epidemic to start before taking any action. This has the effect of decreasing the effectiveness of activities such as larval control, while allowing the public health official to appear responsive to the increase in disease. If the decisionmaker's incentives lie more with public opinion than in preventing disease, it may be difficult to put predictive information to use, even if it is perfectly accurate.

Furthermore, larval control efforts typically rely on at least some cooperation from the public. Therefore, the efficacy of an early warning system for dengue faces an additional challenge in that the forecasting information must be heeded not only by the public health official but also by the community.

Our analysis is also limited by the use of data from Puerto Rico's surveillance system, a system that is not available to many countries with endemic dengue. Using data from Puerto Rico allows for the possibility of generating some estimates on the costs of the disease. Based on these findings, we have provided some indication of what cost-effective programs may look like in Puerto Rico. However, our analysis suggests that other countries are likely to benefit from developing surveillance systems that will allow for some type of early warning signal, even if it is only known seasonal increases in transmission combined with recent epidemic history. An important point of this analysis is that early warning systems have much to add, even if they are not $100 \%$ accurate.

The utilization of an early warning system to reduce dengue transmission

\section{REFERENCES}

in Puerto Rico: 1984-1994. Am J Trop Med Hyg 1998;59(2):265-271.

3. Gubler DJ, Meltzer M. Impact of dengue/ dengue hemorrhagic fever on the developing world. Adv Virus Res 1999;53:3570.

4. Gubler DJ, Clark GG. Community involvement in the control of Aedes aegypti. Acta Trop 1996;61(2):169-179. will not be straightforward. If these warnings are to be put to use to avoid dengue epidemics, there must be a coordinated transfer of information from the organization that provides surveillance to the organizations or individuals that can make the decision about whether an early warning is warranted. Once that point is reached, the success of this information will depend on the ability of municipality leaders to acquire the resources necessary for emergency larval control, to identify the individuals to carry out the work, and to garner the support of the people living in the municipality. Such understanding of the coordination of the different players in such an effort may be particularly important in bridging the gap between research and application.

The aims of this analysis are to provide some estimate of the cost-effectiveness of larval control programs, and to show that early warning systems can improve the cost-effectiveness of intensive larval control programs. This analysis is not intended to provide an incentive to forego measures to reduce dengue transmission. Rather, it is to highlight the importance of selecting larval control programs that have some degree of effectiveness, and to provide incentives for the incorporation of types of early warning systems, which may have substantial benefits even if they are not always $100 \%$ accurate.

Acknowledgments. In writing this paper, we have benefited from the comments of José G. Rigau-Pérez and two anonymous referees. The views in this paper, and any remaining errors or oversights, are our own.
5. Lok CK, Bos R. Control of dengue vectors: Singapore's success story. World Health Forum 1987;8(1):101-104.

6. Gubler DJ, Clark GG. Community-based integrated control of Aedes aegypti: a brief overview of current programs. Am J Trop Med Hyg 1994;50(6 Suppl):50-60.

7. Leontsini E, Gil E, Kendall C, Clark GG. Effect of a community-based Aedes aegypti control 
programme on mosquito larval production sites in El Progreso, Honduras. Trans R Soc Trop Med Hyg 1993;87(3):267-271.

8. Lloyd LS, Winch P, Ortega-Canto J, Kendall C. Results of a community-based Aedes aegypti control program in Merida, Yucatan, Mexico. Am J Trop Med Hyg 1992;46(6):635-642.

9. Focks DA, Daniels E, Haile DG, Keesling JE. A simulation model of the epidemiology of urban dengue fever: literature analysis, model development, preliminary validation, and samples of simulation results. Am J Trop Med Hyg 1995;53(5):489-506.

10. Focks DA, Haile DG, Daniels E, Mount GA. Dynamic life table model for Aedes aegypti (Diptera: Culicidae): analysis of the literature and model development. J Med Entomol 1993;30(6):1003-1017.

11. Focks DA, Haile DG, Daniels E, Mount GA. Dynamic life table model for Aedes aegypti (Diptera: Culicidae): simulation results and validation. J Med Entomol 1993;30(6):10181028.

12. United States of America, Census Bureau. Population estimates for Puerto Rico and the outlying areas: 1980 to 1988. Washington, D.C.: Department of Commerce; 1989.

13. United States of America, Census Bureau. 1990 census of population: general popula- tion characteristics, Puerto Rico. Washington D.C.: Department of Commerce; 1992.

14. United States of America, Census Bureau 1990 census of population and housing: population and housing unit counts, Puerto Rico. Washington, D.C.: Department of Commerce; 1993.

15. Dechant EJ, Rigau-Pérez JG. Hospitalizations for suspected dengue in Puerto Rico, 19911995: estimation by capture-recapture methods. The Puerto Rico Association of Epidemiologists. Am J Trop Med Hyg 1999;61(4) 574-578.

16. Von Allmen SD, López-Correa RH, Woodall JP, Morens DM, Chiriboga J, Casta-Vélez A Epidemic dengue fever in Puerto Rico, 1977: a cost analysis. Am J Trop Med Hyg 1979;28(6) 1040-1044.

17. Torres MI. Impact of an outbreak of dengue fever: a case study from rural Puerto Rico. Human Organization 1997;56(1):19-27.

18. Rigau-Pérez JG, Ayuso-Lamadrid A, Wolff DR Reiter P, Kuno G. Dengue severity throughout seasonal changes in incidence in Puerto Rico, 1989-1992. The Puerto Rico Association of Epidemiologists. Am J Trop Med Hyg 1994 51(4):408-415.

19. Viscusi WK. The value of risks to life and health. J Econ Lit 1993;31:1912-1946.
20. United States of America, Social Security Administration. Earnings and employment data for workers covered under Social Security, by state and county. Washington, D.C.: Social Security Administration; 1994.

21. Rigau-Pérez JG, Millard PS, Walker DR, Deseda CC, Casta-Vélez A. A deviation bar chart for detecting dengue outbreaks in Puerto Rico. Am J Public Health 1999;89(3): 374-378.

22. Gubler DJ, Casta-Valez A. A program for prevention and control of epidemic dengue and dengue hemorrhagic fever in Puerto Rico and the U.S. Virgin Islands. Bull Pan Am Health Organ 1991;25(3):237-247.

23. Focks DA, Brenner RJ, Hayes J, Daniels E. Transmission thresholds for dengue in terms of Aedes aegypti pupae per person with discussion of their utility in source reduction efforts. Am J Trop Med Hyg 2000;62(1):11-18.

24. Glantz MH. Consequences and responsibilities in drought forecasting: the case of Yakima, 1977. Water Resour Res 1982;18(1):3-13.

Manuscript received 22 August 2002. Revised manuscript accepted for publication on 20 February 2003

RESUMEN Objetivos. En los últimos 20 años, las medidas para evitar las epidemias de dengue se han centrado en el control de las larvas del mosquito Aedes aegypti, el principal vector de los virus del dengue. Existe consenso acerca de que el control de las larvas de mosquito es el método más prometedor para reducir las epidemias de dengue, a pesar de que su eficacia real aún se desconoce y constituye un elemento de gran incertidumbre. El objetivo de la presente investigación fue evaluar la rentabilidad de los programas de emergencia para el control de larvas, dirigidos a reducir la transmisión del dengue en la isla caribeña de Puerto Rico, y desarrollar una guía que permita decidir si se deben realizar o no intervenciones de emergencia para el control de larvas como respuesta rápida.

Diseño y métodos. Los datos sobre la transmisión del dengue y su probable impacto en Puerto Rico fueron utilizados a manera de estudio de caso para desarrollar una guía de intervención. Se simuló una distribución de los valores de impacto económico utilizando para ello los datos disponibles de morbilidad y variables económicas, entre ellas el costo del tratamiento de los pacientes y los costos de oportunidad que representan los salarios perdidos. El éxito de las intervenciones de control de larvas fue evaluado mediante dos parámetros: (1) el costo de una intervención y (2) la reducción esperada de casos reportados de dengue. Adicionalmente, se examinó cómo esta guía podría variar con un sistema de alerta rápida que avise de posibles brotes de dengue. Resultados. En Puerto Rico, los programas de control de larvas que prevean reducir la transmisión en $50 \%$ y que cuesten menos de US\$2,50 por persona serán rentables. Los programas que cuesten más por persona pero que logren mayores reducciones de la transmisión de la enfermedad pueden aun ser rentables. Los sistemas de alerta rápida, incluso si su nivel de exactitud es bajo, pueden hacer que más programas para el control de larvas sean rentables. Por ejemplo, podría esperarse que con un sistema de alerta rápida, un programa que reduzca la transmisión del dengue en menos de $50 \%$ y cuyo costo sea menor de US\$ 4,50 por persona, sea rentable.

Conclusiones. Guías como esta, desarrollada para Puerto Rico, pueden ayudar a las autoridades sanitarias a decidir si deben dedicar o no recursos a un programa de control de larvas para reducir la transmisión del dengue. El número de intervenciones rentables para el control de larvas puede aumentar mediante la implementación de un sistema de alerta rápida que facilite información acerca de posibles brotes, aun cuando esta sea escasa. 